\title{
Accurate Wide Modulus Range Nanomechanical Mapping of Ultrathin Interfaces with Bimodal Atomic Force Microscopy
}

\author{
Victor G. Gisbert and Ricardo Garcia* \\ Instituto de Ciencia de Materiales de Madrid, CSIC, c/ Sor Juana Inés de la Cruz 3, 28049 Madrid, \\ Spain
}

\begin{abstract}
Nanoscale determination of the mechanical properties of interfaces is of paramount relevance in materials science and cell biology. Bimodal AFM is arguably the most advanced nanoscale method to map the elastic modulus of interfaces. Simulations, theory and experiments have validated bimodal AFM measurements on thick samples (from $\mu \mathrm{m}$ to $\mathrm{mm}$ ). However, the bottom-effect artifact, this is, the influence of the rigid support on the determination of the Young's modulus questions its accuracy for ultrathin materials and interfaces (1-15 nm). Here, we develop a bottom-effect correction method that yields the intrinsic Young's modulus value of a material with independence of its thickness. Experiments and numerical simulations validate the accuracy of the method for a wide range of materials (1 MPa-100 GPa). Otherwise, the Young's modulus of an ultrathin material might be overestimated by a 10 -fold factor.
\end{abstract}

\section{KEYWORDS}

Nanomechanics, bimodal AFM, elastic modulus, ultrathin layers, lipid bilayers 


\section{INTRODUCTION}

Nanoscale mechanical property mapping is one of the key achievements of atomic force microscopy (AFM) ${ }^{1-5}$ Nanomechanical mapping is applied to facilitate the development or to improve the characterization of materials and interfaces. ${ }^{6-15}$ Nanomechanical measurements are widely used in mechanobiology to reveal how elastic and viscoelastic properties of proteins, cells and the extracellular matrix influence biological processes. ${ }^{16-22}$

A variety of AFM-based methods have been developed over the last 25 years to map mechanical properties at the nanoscale. Among them, force volume ${ }^{1,3-4}$, AFM phase imaging ${ }^{2}$, bimodal $\mathrm{AFM}^{4,23}$, torsional harmonics, ${ }^{24-25}$ contact resonance ${ }^{26-28}$ or multifrequency AFM. ${ }^{29}$ The accuracy of some of those methods on thick samples, notably the two most widely applied, force volume (force-distance curve methods) and bimodal AFM have been validated by performing comparisons with macroscopic techniques, finite-element methods and numerical simulations. However, some key applications in materials science and mechanobiology might involve the nanomechanical characterization of ultrathin materials $(1-20 \mathrm{~nm})$ such as nanostructured materials, 2D materials, lipid membranes, DNA or proteins deposited on rigid supports.

Dimitriadis, Chadwick and co-workers ${ }^{30}$ showed that the elastic modulus of the rigid support influences the AFM measurement on cells. This finding led to the so-called bottom effect artifact which, in turn, have stimulated different correction models to address the determination of elastic and viscoelastic properties measured by force volume (AFM-based force-distance curves)..$^{31-34}$ Bottom-effect corrections were also proposed for multilayer materials. ${ }^{33,}{ }^{35-37}$ Several of the corrections were numerical solutions which were difficult to implement to process AFM experiments.

Bimodal AFM is arguably the fastest and most versatile method to map nanomechanical properties of surfaces and interfaces. ${ }^{23,38}$ It has been applied to characterize the elastic properties of polymers, ${ }^{39-40}$ hybrid materials, ${ }^{41}$ inorganic and organic crystals, ${ }^{42-43}$ self-assembled 
monolayers, ${ }^{44}$ lipid membranes, ${ }^{45}$ DNA,${ }^{41,46}$ proteins, ${ }^{47-50}$ and cells. ${ }^{51}$ It combines high-spatial resolution (angstrom or nanoscale) with quantitative accuracy on the determination of the Young's modulus. Recently, the method has been extended to measure adhesion forces at the nanoscale..$^{52}$

A variety of theories and simulations have supported and/or demonstrated the capabilities of bimodal AFM. ${ }^{53-63}$ Currently, it is the only method that provides nanomechanical maps at 5 frames per second (high speed). ${ }^{50}$ Despite its widespread use, the accuracy of the bimodal nanomechanical measurements performed on ultrathin materials might be jeopardized by the bottom-effect artifact. ${ }^{4,30}$

Here, we develop a bottom-effect correction method for bimodal elastic property measurements performed on ultrathin layers and nanostructured materials. Numerical simulations show that the analytical expression enable to recover the Young's modulus of ultrathin layers with a relative error below $5 \%$ over a 5 -order of magnitude modulus range (1 MPa-100 GPa). The method is applied to determine Young's modulus of supported lipid bilayers immersed in liquid. Bimodal AFM provided a Young's modulus value of $13 \mathrm{MPa}$ which was independent on the number of lipid bilayers. Force volume measurements performed on the same sample provided a similar value.

\section{RESULTS AND DISCUSSION}

Figure 1 shows a scheme of bimodal AFM nanomechanical measurement performed on ultrathin materials. Figure 1a shows the tip-layer-solid support interface while Fig. 1b describes some parameters of bimodal AFM. Specifically, we have applied the bimodal AM-FM configuration. In this configuration, the amplitude of the first mode is kept constant while the phase shift of the second mode is locked at $90^{\circ}$ with respect to its driving force..$^{48,64}$ 
Bottom effect correction theory for bimodal AFM. In bimodal AFM, the procedure to determine elastic modulus from the observables is well established. It involves the application of the virial theorem to excited modes. ${ }^{48,53}$ This leads to two independent equations, one for each excited mode,

$V_{i}=\left\langle F_{t s} z_{i}\right\rangle=\frac{1}{T} \int_{0}^{T} F_{t s}(z) z_{i} d t$

where $z_{i}$ are the deflection of the excited modes and $z$ is the total cantilever deflection $\left(z \approx z_{1}+z_{2}\right)$. In many situations of interest, $T$ is well approximated by the period of the first eigenmode $(T \approx$ $\left.T_{1}\right) .{ }^{50,52}$ The above equation can be solved by two independent approaches. First, by using integrating the equation of motion of the excited modes. It has been shown that for $\left(A_{01}>>A_{02}\right)^{48,53}$

$V_{1 m}=\frac{k_{1} A_{1} A_{01}}{2 Q_{1}} \cos \phi_{1}$

$V_{2 m}=\frac{k_{2} \Delta f_{2}}{f_{2}} A_{2}^{2}$

We have used the subscript $m$ to indicate that the virials are directly determined (measured) from the observables $\left(A_{1}, A_{2}, \phi_{1}, \Delta f_{2}\right)$. In the above equations $k_{1}, Q_{1}, A_{1}$ and $A_{0}$ are respectively the force constant, quality factor, instantaneous amplitude and free amplitude of the $1^{\text {st }}$ eigenmode; $k_{2}, A_{2}$, $f_{2}$ and $\Delta f_{2}$ are respectively the force constant, instantaneous amplitude, resonant frequency and resonant frequency shift of the $2^{\text {nd }}$ eigenmode. Second, the virials might be independently determined by solving the integral in eq 1 with a $F_{t s}$ force given by a contact mechanics model.

In a previous contribution, ${ }^{48}$ we deduced an analytical expression to determine the Young's modulus $E_{\mathrm{s}}$ based on a semi-infinite model. The expression was deduced by assuming that the force exerted by a paraboloid of radius $R$ on a material (with independence of its thickness) was described as a function of the indentation $I$ by the Hertz (Sneddon-paraboloid) model ${ }^{65}$ 
In this approximation, the effective Young's modulus $E$ of the interface is given by

$E($ semi $)=\frac{1}{I^{2}} \frac{k_{1}}{Q_{1}}\left(\frac{2 A_{1}\left(A_{01}^{2}-A_{1}^{2}\right)}{R}\right)^{1 / 2}$

with

$\frac{1}{E}=\frac{1-v_{s}^{2}}{E_{S}}+\frac{1-v_{t i p}^{2}}{E_{t i p}}$

$s$ stands for sample and $v_{t i p}, v_{s}$ is the Poisson's ratio of the tip and the sample. Here, we aim to determine the Young's modulus of a layer of thickness $h$ deposited on a rigid material. For this interface, the force exerted by a paraboloid as function of the indentation is determined by the expression deduced by Garcia and Garcia ${ }^{31}$

$F_{t s}=F_{\text {Hertz }}\left[1+F_{b e c}(a / h)\right]$

$F_{b e c}=\left(1.133\left(\frac{\sqrt{R I}}{h}\right)+1.497\left(\frac{\sqrt{R I}}{h}\right)^{2}+1.469\left(\frac{\sqrt{R I}}{h}\right)^{3}+0.755\left(\frac{\sqrt{R I}}{h}\right)^{4}\right)$

where $a$ is the contact radius

$a=\sqrt{R I}$

By introducing eq. 7 into eq. 1, the expressions for $V_{l}(E, I)$ and $V_{2}(E, I)$ are obtained. These equations are detailed in the Supporting information. Those expressions in combination with equations 2 and 3 lead to a systems of equations 
$\left\{\begin{array}{l}V_{1}(E, I)-V_{1 m}=0 \\ V_{2}(E, I)-V_{2 m}=0\end{array}\right.$

The solution of the above system provides an analytical and closed-form expression to determine the Young's modulus of a layer with bimodal AFM,

$E($ layer $)=\frac{\sqrt{8}}{I^{2} \sqrt{R A_{1}}} \frac{V_{1 m}}{1+1.03 \frac{\sqrt{R I}}{h}+1.25\left(\frac{\sqrt{R I}}{h}\right)^{2}+1.14\left(\frac{\sqrt{R I}}{h}\right)^{3}+0.55\left(\frac{\sqrt{R I}}{h}\right)^{4}}$

We have omitted some intermediate steps ${ }^{66}$ which are detailed in the SI.

Accuracy assessment. To assess the accuracy and modulus range to determine the elastic modulus of ultrathin layers with bimodal AFM, we have performed simulations, experiments and comparisons with force-distance curve measurements.

Simulations. We have used an open-source dynamic AFM code (dForce) to simulate bimodal AFM operation ${ }^{67}$ Figure 2 shows the dependence of the Young's modulus of the sample (layer) $\left(E_{s}=10 \mathrm{MPa}\right)$ as a function of the indentation, thickness of the layer and tip's radius. The figure compares the values obtained by using the expression for a semi-infinite material (eq. 5) with those given by the expression deduced for a finite thickness layer (eq 11). Three different thickness were contemplated, $7 \mathrm{~nm}, 14 \mathrm{~nm}$ and semi-infinite. The first two values were chosen to match the thickness of single and double DCCP lipid bilayers.

The bottom effect correction provides values that coincide or are very close with the nominal value (10 MPa) (Fig. 2a). The semi-infinite expression tends to overestimate the Young's modulus. The overestimation increases with the indentation and could lead to significant errors (several-fold factors). We remark that the stability and accuracy of bimodal AFM measurements 
depend on the values of the free and set point amplitudes ( $1^{\text {st }}$ mode). Therefore, a minimum indentation is needed to probe the elastic response of a material. The value depends on the elastic modulus. For a $10 \mathrm{MPa}$ material, the minimum indentation to get a stable bimodal AFM operation is about $0.5 \mathrm{~nm}$.

Figure $2 \mathrm{~b}$ shows Young's modulus dependence on the indentation for different tip's radii $(R=1$, 2, 4, 8 and 10). In bimodal AFM, the maximum indentation is increased by decreasing the amplitude ratio $\left(A_{1} / A_{01}\right)$. The Young's modulus obtained by using the bottom-effect correction (eq 11) shows a region of operational parameters where the Young's modulus might be determined with an error below $10 \%$. This requirement is very hard to achieve if the layer is treated as semi-infinite material (eq. 5). In fact, it only happens for the sharper tip $(R=1 \mathrm{~nm})$ and within a very small range of indentations $(0.5-0.8 \mathrm{~nm})$.

The results presented in Figure 2 underline a counter-intuitive result in nanomechanical mapping of ultrathin materials. All other factors equal, the error introduced by using a semi-infinite model to determine the elastic modulus increases by increasing the tip radius. This happens because the bottom effect artifact depends on the contact area, which for the same indentation, increases with the tip's radius.

Bimodal AFM nanomechanical maps on lipid bilayers. To illustrate the process of recovering the Young's modulus of an ultrathin soft material, we have performed experiments on lipid layers. This system enables to prepare layers of different thickness (multiples of the bilayer thickness). This feature provides a convenient experimental model to test the validity of the theory. Chiodini et al. used lipid bilayers to apply bottom effect corrections to force-distance curves. ${ }^{34}$ Lipid bilayers are of relevance in cell biology and biotechnology. ${ }^{68-70}$

Figure 3 shows the topography (Fig. 3a) and Young's modulus maps (Fig. 3b-c) of a region that includes single lipid bilayer and double lipid bilayers domains. Figure $3 b$ shows the elastic 
modulus map obtained by using the finite thickness theory (bottom effect correction) while Fig. $3 \mathrm{c}$ shows the map obtained by using the semi-infinite theory. The cross-sectional profiles across the lines marked in the maps together with the histograms taken over the whole region are shown in the bottom panels. The height of the single and double bilayers were, respectively, 6 and 12 $\mathrm{nm}$ (average values). The Young's modulus determined by using a semi-infinite model depends on the thickness (40 MPa for a single bilayer and $22 \mathrm{MPa}$ for a double bilayer). This no longer the case when the bottom-effect correction is applied. The first bilayer gives a modulus of 11.6 MPa while the $2^{\text {nd }}$ bilayer gives $11.9 \mathrm{MPa}$. The difference is within the $5 \%$ relative error.

Another interesting observation comes from analysing the dependence of the force on the layer thickness, indentation and tip's radius (Figure 4). For the same indentation, the peak (maximum) force applied on a layer decreases with the layer thickness (Fig. 4a) and increases with the tip's radius (Fig. 4b). Figure 4c shows the experimental values of the indentation (Fig. 4c) and the maximum force (Fig. 4d) exerted on the lipid domains (Fig. 3). For the same experimental conditions, the thinner the layer the larger the force and conversely the smaller the indentation. These curves indicate that influence of the rigidity of the solid support on the force is an intrinsic property of the interface, as such, it is unavoidable. By knowing the layer thickness, the bottomeffect correction manages to recover the correct Young's modulus of the material with independence of its thickness.

To check the consistency of the above value, we have also determined the Young's modulus of the lipid bilayers domains by acquiring force-distance curves. Figure 5 shows the box-plots of the Young's modulus obtained from bimodal (Fig. 5a) and force-volume (Fig. 5b) maps of the same a region of the sample. Again, the use of semi-infinite model and theory provides values which depend on the layer thickness. This artifact is corrected by applying the bottom-effect correction. Significantly, both methods provided similar values for the lipid bilayer with independence of the number of bilayers when bottom effect corrections were applied. 
To generalize the results, we have performed simulations for materials ranging from $1 \mathrm{MPa}$ to $100 \mathrm{GPa}$. Figure 6 shows that the use of a semi-infinite model might lead to a 10 -fold overestimation of the Young's modulus of an ultrathin material. On the other hand, the bottomeffect correction recovers the proper Young's modulus with independence of the layer thickness. The simulations illustrate another counter-intuitive feature of the bottom-effect artifact. For ultrathin layers, say below $15 \mathrm{~nm}$, the bottom-effect artifact is also present in a $10 \mathrm{GPa}$ layer. In the bottom-effect correction, the influence of the rigid support is weighted by the $a / h$ ratio.

We remark that the bottom-effect corrections are essential to provide accurate quantitative elastic property values of materials, specifically of ultrathin materials. However, many bimodal AFM applications aim to enhance spatial resolution or material contrast. ${ }^{71-78}$ Those applications do not require any bottom effect correction.

\section{CONCLUSIONS}

The force exerted by a tip on an ultrathin material deposited on a rigid support bears the influence of the mechanical response of the support. This is an intrinsic property of the interaction and the structure of the interface. Therefore, to determine a material property of an ultrathin material with bimodal AFM requires to process the instrument observables with a finite thickness model that accounts for the bottom effect artifact.

Bimodal AFM is arguably the most advanced method to determine the nanomechanical properties of materials and interfaces. We have developed a model that enables to recover the intrinsic Young's modulus of a material deposited on a solid support with independence of its thickness by bimodal AFM.

We demonstrate that bimodal AFM provides accurate Young's modulus values of ultrathin layers (1-15 nm). In fact, those values are independent of the layer thickness. The model was validated 
for wide range of materials (1 MPa-100 GPa). The validity and accuracy of the bimodal method was tested by simulations, experiments and comparison with force volume measurements.

We remark that the application of conventional contact mechanics models, this is, those which consider the material as semi-infinite leads to overestimate the Young's modulus of an ultrathin material by a factor 10-fold or larger. Contrary to common wisdom, the error in the estimation of the Young's modulus might be significant even for moderate to hard ultrathin materials (10-100 GPa). This might happen whenever the tip-layer contact radius is comparable to the layer thickness.

\section{MATERIALS AND METHODS}

Bimodal AFM experiments. The bimodal AFM measurements were performed in the amplitude modulation-frequency modulation (AM-FM) configuration. ${ }^{48,64}$ An introduction to amplitude and frequency modulation modes is provided in ref. 79. The experiments were performed with a Cypher VRS (Asylum Research Inc.). The bimodal AFM observables were processed with a custom-made software. The experiments were conducted in liquid (see below). USC-F0.3-k0.3 microcantilevers (NanoAndMore, Germany) with $f_{1}=366 \mathrm{kHz}, k_{1}=0.332 \mathrm{~N} \mathrm{~m}-1, Q_{1}=1.1, f_{2}=$ $1621 \mathrm{kHz}, k_{2}=6.52 \mathrm{~N} \mathrm{~m}^{-1}$ were used to image the lipid bilayer. Proper calibration of the force constants of the excited modes $\left(1^{\text {st }} \text { and } 2^{\text {nd }}\right)^{80}$ is essential for accurate nanomechanical mapping. The tips of USC-F0.3-k0.3 cantilevers are made of diamond-like carbon. To deduce the Young's modulus of the lipid bilayers we assumed $v_{\text {tip }}=0.2$ and $E_{t i p}=400 \mathrm{GPa}$ while the Poison ratio for the lipid bilayer was 0.3 . We have applied the method developed by Labuda et al. for calibrating higher eigenmodes. ${ }^{81}$ Typical parameters for bimodal AFM experiments on lipid layers were $A_{01}$ $=2.0 \mathrm{~nm}, A_{1}=1.8 \mathrm{~nm}, A_{2}=0.1 \mathrm{~nm}, F_{\max }=250 \mathrm{pN}, I_{\max }=1.6 \mathrm{~nm}$. 
Bimodal AFM bottom-effect theory. The intermediate steps to deduce the relationship between the Young's modulus and the bimodal observables are presented in the Supporting Information.

Force-distance curves. Force-distance curves (FDC) on lipid bilayers were obtained with the same cantilever right after performing the bimodal AFM measurements. FDCs were recorded at $2 \mathrm{~Hz}$. The indentation was stopped when the force reached $240 \mathrm{pN}$. The force-distance curves were fitted with the bottom effect correction deduced for paraboloid tip. ${ }^{31}$

Lipid bilayers. Dipalmitoylphosphatidylcholine (DPPC) powder (Avanti - 850355P-200MG 16:0 PC (DPPC)) was dissolved in Chloroform/Ethanol 2/1 (vol/vol) to a final concentration of 1 $\mathrm{mM}$, evaporated under a nitrogen flow and dried on a desiccator for 2 hours. ${ }^{68}$ The dried lipids were resuspended in buffer $\left(10 \mathrm{mM}\right.$ tris, $100 \mathrm{mM} \mathrm{NaCl}, 3 \mathrm{mM} \mathrm{CaCl}_{2}, \mathrm{pH}$ 7.4) to a final lipid concentration of $1 \mathrm{mM}$. The solution was sonicated 5 times for 2 minutes while keeping the suspension in an ice bath to obtain the small unilamellar vesicles (SUVs) suspension. $50 \mu 1$ of the suspension was deposited into a freshly cleaved muscovite mica disk (Grade V-1, Alpha Biotech Ltd.) and incubated for 60 minutes in a closed container to avoid evaporation. To favor the formation of a double bilayer ${ }^{69} 50 \mu 1$ of the DPPC solution was added every 15 minutes. After deposition the sample was carefully rinsed with Imaging buffer $(10 \mathrm{mM}$ Tris, $100 \mathrm{mM} \mathrm{NaCl}, \mathrm{pH}$ 7.4). The sample was placed inside a fluid chamber. AFM imaging started without further delays. The measurements were performed at a temperature of $22{ }^{\circ} \mathrm{C}$.

\section{ASSOCIATED CONTENT}

\section{Supporting Information}

The Supporting Information is available free of charge at DOI: 
Additional force volume AFM maps of the lipids bilayers, additional details of simulations and theory.

Figure S1: Force volume maps of single and double lipid bilayers (DPPC).

Figure S2: Numerical simulations of bimodal AFM of a material with different thicknesses

Figure S3: Numerical simulations of bimodal AFM of a finite thick layer for different tip radius

\section{AUTHOR INFORMATION}

Corresponding Author

*Email: r.garcia@csic.es.

\section{AUTHOR CONTRIBUTIONS}

V.G.G deduced the analytical expressions, and performed the experiments and simulations.

R.G. designed the simulations, the experiments and wrote the manuscript. Both authors discussed the results and revised the manuscript.

\section{Note}

The authors declare the following competing financial interest: R.G. is inventor of several patents on bimodal AFM methods.

\section{ACKNOWLEDGEMENTS}


Financial support from the Ministerio de Ciencia e Innovación (PID2019-106801GB-I00), CSIC

202050E013, Comunidad de Madrid S2018/NMT-4443 (Tec4Bio-CM) are acknowledged.

\section{REFERENCES}

(1) Heinz, W. F.; Hoh, J. H. Spatially Resolved Force Spectroscopy of Biological Surfaces Using the Atomic Force Microscope. Trends in Biotechnology 1999, 17, 143-150.

(2) Garcia, R.; Magerle, R.; Perez, R. Nanoscale Compositional Mapping with Gentle Forces. Nat. Mater. 2007, 6, 405-411.

(3) Dufrêne, Y. F.; Martínez-Martín, D.; Medalsy, I.; Alsteens, D.; Müller, D. J.

Multiparametric Imaging of Biological Systems by Force-Distance Curve-Based AFM. Nat. Methods 2013, 10, 847-854.

(4) Garcia, R. Nanomechanical Mapping of Soft Materials with the Atomic Force Microscope: Methods, Theory and Applications. Chem. Soc. Rev. 2020, 49, 5850-5884.

(5) Stan, G.; King, S. W. Atomic Force Microscopy for Nanoscale Mechanical Property Characterization. Journal of Vacuum Science \& Technology B 2020, 38, 060801.

(6) Collinson, D. W.; Sheridan, R. J.; Palmeri, M. J.; Brinson, L. C. Best Practices and Recommendations for Accurate Nanomechanical Characterization of Heterogeneous Polymer Systems with Atomic Force Microscopy. Progress in Polymer Science 2021, 119, 101420.

(7) Wang, D.; Russell, T. P. Advances in Atomic Force Microscopy for Probing Polymer Structure and Properties. Macromolecules 2018, 51, 3-24.

(8) Nguyen, H. K.; Ito, M.; Nakajima, K. Elastic and Viscoelastic Characterization of Inhomogeneous Polymers by Bimodal Atomic Force Microscopy. Jpn. J. Appl. Phys. 2016, 55, 08NB06.

(9) Cortelli, G.; Patruno, L.; Cramer, T.; Murgia, M.; Fraboni, B.; de Miranda, S. Atomic Force Microscopy Nanomechanics of Hard Nanometer-Thick Films on Soft Substrates: Insights into Stretchable Conductors. ACS Appl. Nano Mater. 2021, 4, 8376-8382.

(10) Li, Y.; Yu, C.; Gan, Y.; Jiang, P.; Yu, J.; Ou, Y.; Zou, D.-F.; Huang, C.; Wang, J.; Jia, T.; Luo, Q.; Yu, X.-F.; Zhao, H.; Gao, C.-F.; Li, J. Mapping the Elastic Properties of TwoDimensional MoS2 via Bimodal Atomic Force Microscopy and Finite Element Simulation. npj Comput. Mater. 2018, 4, 49.

(11) Lu, J.-Y.; Olukan, T.; Tamalampudi, S. R.; Al-Hagri, A.; Lai, C.-Y.; Ali Al Mahri, M.; Apostoleris, H.; Almansouri, I.; Chiesa, M. Insights into Graphene Wettability Transparency by Locally Probing Its Surface Free Energy. Nanoscale 2019, 11, 7944-7951.

(12) Magerle, R.; Dehnert, M.; Voigt, D.; Bernstein, A. Nanomechanical 3D Depth Profiling of Collagen Fibrils in Native Tendon. Anal. Chem. 2020, 92, 8741-8749.

(13) Fortes Brollo, M. E.; Domínguez-Bajo, A.; Tabero, A.; Domínguez-Arca, V.; Gisbert, V.; Prieto, G.; Johansson, C.; Garcia, R.; Villanueva, A.; Serrano, M. C.; Morales, M. del P. Combined Magnetoliposome Formation and Drug Loading in One Step for Efficient Alternating Current-Magnetic Field Remote-Controlled Drug Release. ACS Appl. Mater. Interfaces 2020, $12,4295-4307$. 
(14) Giridharagopal, R.; Flagg, L. Q.; Harrison, J. S.; Ziffer, M. E.; Onorato, J.; Luscombe, C. K.; Ginger, D. S. Electrochemical Strain Microscopy Probes Morphology-Induced Variations in Ion Uptake and Performance in Organic Electrochemical Transistors. Nat. Mater. 2017, 16, 737-742.

(15) López-Polín, G.; Gómez-Navarro, C.; Parente, V.; Guinea, F.; Katsnelson, M. I.; PérezMurano, F.; Gómez-Herrero, J. Increasing the Elastic Modulus of Graphene by Controlled Defect Creation. Nat. Phys. 2015, 11, 26-31.

(16) Chaudhuri, O.; Cooper-White, J.; Janmey, P. A.; Mooney, D. J.; Shenoy, V. B. Effects of Extracellular Matrix Viscoelasticity on Cellular Behaviour. Nature 2020, 584, 535-546.

(17) X. Meng, H. Zhang, J. Song, X. Fan and L. Sun, Broad Modulus Range Nanomechanical Mapping by Magnetic-Drive Soft Probes, Nat. Commun. 2017, 8, 1944.

(18) Medalsy, I.; Hensen, U.; Muller, D. J. Imaging and Quantifying Chemical and Physical Properties of Native Proteins at Molecular Resolution by Force-Volume AFM. Angew. Chem. Int. Ed. 2011, 50, 12103-12108.

(19) Preiner, J.; Horner, A.; Karner, A.; Ollinger, N.; Siligan, C.; Pohl, P.; Hinterdorfer, P. High-Speed AFM Images of Thermal Motion Provide Stiffness Map of Interfacial Membrane Protein Moieties. Nano Lett. 2015, 15, 759-763.

(20) Guerrero, C. R.; Garcia, P. D.; Garcia, R. Subsurface Imaging of Cell Organelles by Force Microscopy. ACS Nano 2019, 13, 9629-9637.

(21) Mandriota, N.; Friedsam, C.; Jones-Molina, J. A.; Tatem, K. V.; Ingber, D. E.; Sahin, O. Cellular Nanoscale Stiffness Patterns Governed by Intracellular Forces. Nat. Mater. 2019, 18, 1071-1077.

(22) Sun, Y.; Vu, L. H.; Chew, N.; Puthucheary, Z.; Cove, M. E.; Zeng, K. A Study of Perturbations in Structure and Elastic Modulus of Bone Microconstituents Using Bimodal Amplitude Modulated-Frequency Modulated Atomic Force Microscopy. ACS Biomater. Sci. Eng. 2019, 5, 478-486.

(23) Rodriguez, T. R.; Garcia, R. Compositional Mapping of Surfaces in Atomic Force Microscopy by Excitation of the Second Normal Mode of the Microcantilever. Appl. Phys. Lett. 2004, 84, 449-451.

(24) Sahin, O.; Magonov, S.; Su, C.; Quate, C. F.; Solgaard, O. An Atomic Force Microscope Tip Designed to Measure Time-Varying Nanomechanical Forces. Nat. Nanotech 2007, 2, 507514.

(25) Liu, L.; Li, Q.; Zhang, S.; Wang, X.; Hoffmann, S. V.; Li, J.; Liu, Z.; Besenbacher, F.; Dong, M. Identification of a Novel Parallel $\beta$-Strand Conformation within Molecular Monolayer of Amyloid Peptide. Adv. Sci. 2016, 3, 1500369.

(26) U. Rabe; S. Amelio; M. Kopycinska; S. Hirsekorn; M. Kempf; M. Göken; W. Arnold. Imaging and Measurement of Local Mechanical Material Properties by Atomic Force Acoustic Microscopy. Surf. Interface Anal. 2002, 33, 65-70.

(27) Killgore, J. P.; Del Rio, F. W. Contact Resonance Force Microscopy for Viscoelastic Property Measurements: From Fundamentals to State-of-the-Art Applications. Macromolecules 2018, 51, 6977-6996. 
(28) Sharahi, H. J.; Janmaleki, M.; Tetard, L.; Kim, S.; Sadeghian, H.; Verbiest, G. J. Acoustic Subsurface-Atomic Force Microscopy: Three-Dimensional Imaging at the Nanoscale. J. Appl. Phys. 2021, 129, 030901.

(29) Garcia, R.; Herruzo, E. T. The Emergence of Multifrequency Force Microscopy. Nat. Nanotech. 2012, 7, 217-226.

(30) Dimitriadis, E. K.; Horkay, F.; Maresca, J.; Kachar, B.; Chadwick, R. S. Determination of Elastic Moduli of Thin Layers of Soft Material Using the Atomic Force Microscope.

Biophysical Journal 2002, 82, 2798-2810.

(31) Garcia, P. D.; Garcia, R. Determination of the Elastic Moduli of a Single Cell Cultured on a Rigid Support by Force Microscopy. Biophysical Journal 2018, 114, 2923-2932.

(32) Garcia, P. D.; Garcia, R. Determination of the Viscoelastic Properties of a Single Cell Cultured on a Rigid Support by Force Microscopy. Nanoscale 2018, 10, 19799-19809.

(33) Doss, B. L.; Rahmani Eliato, K.; Lin, K.; Ros, R. Quantitative Mechanical Analysis of Indentations on Layered, Soft Elastic Materials. Soft Matter 2019, 15, 1776-1784.

(34) Chiodini, S.; Ruiz-Rincón, S.; Garcia, P. D.; Martin, S.; Kettelhoit, K.; Armenia, I.; Werz, D. B.; Cea, P. Bottom Effect in Atomic Force Microscopy Nanomechanics. Small 2020, 16, 2000269.

(35) Stan, G.; Adams, G. G. Adhesive Contact between a Rigid Spherical Indenter and an Elastic Multi-Layer Coated Substrate. Int. J. Solids Struct. 2016, 87, 1-10.

(36) Onur Sergici, A.; Adams, G. G.; Müftui, S. Adhesion in the Contact of a Spherical Indenter with a Layered Elastic Half-Space. J. Mech. Phys. Solids 2006, 54 (9), 1843-1861.

(37) Constantinescu, A.; Korsunsky, A. M.; Pison, O.; Oueslati, A. Symbolic and Numerical Solution of the Axisymmetric Indentation Problem for a Multilayered Elastic Coating. Int. J. Solids Struct. 2013, 50 (18), 2798-2807.

(38) Garcia, R.; Proksch, R. Nanomechanical Mapping of Soft Matter by Bimodal Force Microscopy. European Polymer Journal 2013, 49, 1897-1906.

(39) Herruzo, E. T.; Perrino, A. P.; Garcia, R. Fast Nanomechanical Spectroscopy of Soft Matter. Nat. Commun. 2014, 5, 3126.

(40) Nguyen, H. K.; Aoki, M.; Liang, X.; Yamamoto, S.; Tanaka, K.; Nakajima, K. Local Mechanical Properties of Heterogeneous Nanostructures Developed in a Cured Epoxy Network: Implications for Innovative Adhesion Technology. ACS Appl. Nano Mater. 2021, Article ASAP. DOI: 10.1021/acsanm.1c02692 (accessed 2021-11-17).

(41) Kocun, M.; Labuda, A.; Meinhold, W.; Revenko, I.; Proksch, R. Fast, High Resolution, and Wide Modulus Range Nanomechanical Mapping with Bimodal Tapping Mode. ACS Nano 2017, 11, 10097-10105.

(42) Trewby, W.; Faraudo, J.; Voïtchovsky, K. Long-Lived Ionic Nano-Domains Can Modulate the Stiffness of Soft Interfaces. Nanoscale 2019, 11, 4376-4384.

(43) Dupont, M. F.; Elbourne, A.; Mayes, E.; Latham, K. Measuring the Mechanical Properties of Flexible Crystals Using Bi-Modal Atomic Force Microscopy. Phys. Chem. Chem. Phys.

2019, 21, 20219-20224. 
(44) Athanasopoulou, E.-N.; Nianias, N.; Ong, Q. K.; Stellacci, F. Bimodal Atomic Force Microscopy for the Characterization of Thiolated Self-Assembled Monolayers. Nanoscale 2018, 10, 23027-23036.

(45) Al-Rekabi, Z.; Contera, S. Multifrequency AFM Reveals Lipid Membrane Mechanical Properties and the Effect of Cholesterol in Modulating Viscoelasticity. Proc. Natl. Acad. Sci. USA 2018, 115, 2658-2663.

(46) Lai, C.-Y.; Santos, S.; Chiesa, M. Systematic Multidimensional Quantification of Nanoscale Systems From Bimodal Atomic Force Microscopy Data. ACS Nano 2016, 10, 62656272 .

(47) Gilbert, J.; Charnley, M.; Cheng, C.; Reynolds, N. P.; Jones, O. G. Quantifying Young's Moduli of Protein Fibrils and Particles with Bimodal Force Spectroscopy. Biointerphases 2017, 12, 041001 .

(48) Amo, C. A.; Perrino, A. P.; Payam, A. F.; Garcia, R. Mapping Elastic Properties of Heterogeneous Materials in Liquid with Angstrom-Scale Resolution. ACS Nano 2017, 11, $8650-8659$.

(49) Benaglia, S.; Gisbert, V. G.; Perrino, A. P.; Amo, C. A.; Garcia, R. Fast and HighResolution Mapping of Elastic Properties of Biomolecules and Polymers with Bimodal AFM. Nat. Protoc. 2018, 13, 2890-2907

(50) Gisbert, V. G.; Benaglia, S.; Uhlig, M. R.; Proksch, R.; Garcia, R. High-Speed Nanomechanical Mapping of the Early Stages of Collagen Growth by Bimodal Force Microscopy. ACS Nano 2021, 15, 1850-1857.

(51) Cartagena-Rivera, A. X.; Wang, W.-H.; Geahlen, R. L.; Raman, A. Fast, Multi-Frequency and Quantitative Nanomechanical Mapping of Live Cells Using the Atomic Force Microscope. Sci. Rep. 2015, 5, 11692.

(52) Rajabifar, B.; Bajaj, A. K.; Reifenberger, R. G.; Proksch, R.; Raman, A. Discrimination of Adhesion and Viscoelasticity from Nanoscale Maps of Polymer Surfaces Using Bimodal Atomic Force Microscopy. Nanoscale 2021, 13, 17428-17441.

(53) Lozano, J. R.; Garcia, R. Theory of Phase Spectroscopy in Bimodal Atomic Force Microscopy. Phys. Rev. B. 2009, 79, 014110.

(54) Solares, S. D.; Chawla, G. Frequency Response of Higher Cantilever Eigenmodes in Bimodal and Trimodal Tapping Mode Atomic Force Microscopy. Meas. Sci. Technol. 2010, 21, 125502 .

(55) Aksoy, M. D.; Atalar, A. Force Spectroscopy Using Bimodal Frequency Modulation Atomic Force Microscopy. Phys. Rev. B 2011, 83, 075416.

(56) Herruzo, E. T.; Garcia, R. Theoretical Study of the Frequency Shift in Bimodal FM-AFM by Fractional Calculus. Beilstein J. Nanotechnol. 2012, 3, 198-206.

(57) Forchheimer, D.; Borysov, S. S.; Platz, D.; Haviland, D. B. Determining Surface Properties with Bimodal and Multimodal AFM. Nanotechnology 2014, 25, 485708.

(58) Lai, C.-Y.; Barcons, V.; Santos, S.; Chiesa, M. Periodicity in Bimodal Atomic Force Microscopy. J. Appl. Phys. 2015, 118, 044905. 
(59) Labuda, A.; Kocuń, M.; Meinhold, W.; Walters, D.; Proksch, R. Generalized Hertz Model for Bimodal Nanomechanical Mapping. Beilstein J. Nanotechnol. 2016, 7, 970-982.

(60) Payam, A. F.; Trewby, W.; Voïtchovsky, K. Simultaneous Viscosity and Density Measurement of Small Volumes of Liquids Using a Vibrating Microcantilever. Analyst 2017, 142, 1492-1498.

(61) Eslami, B.; Damircheli, M. Biharmonic versus Bimodal AFM: Numerical and Experimental Study on Soft Matter. J. Appl. Phys. 2019, 126, 095301.

(62) Harcombe, D. M.; Ruppert, M. G.; Fleming, A. J. A Review of Demodulation Techniques for Multifrequency Atomic Force Microscopy. Beilstein J. Nanotechnol. 2020, 11, 76-91.

(63) Dou, Z.; Qian, J.; Li, Y.; Wang, Z.; Zhang, Y.; Lin, R.; Wang, T. Molecular Dynamics Simulation of Bimodal Atomic Force Microscopy. Ultramicroscopy 2020, 212, 112971.

(64) Chawla, G. \& Solares, S. D. Mapping of Conservative and Dissipative Interactions in Bimodal Atomic Force Microscopy Using Open-Loop and Phase-Locked-Loop Control of the Higher Eigenmode. Appl. Phys. Lett. 2011, 99, 074103.

(65) Sneddon, I. N. The Relation between Load and Penetration in the Axisymmetric Boussinesq Problem for a Punch of Arbitrary Profile. Int. J. Eng. Sci. 1965, 3, 47-57.

(66) Bateman, H.; Erdéllyi, A. Tables of integral transforms 1; McGraw-Hill: New York, 1954

(67) Guzman, H. V.; Garcia, P. D.; Garcia, R. Dynamic Force Microscopy Simulator (dForce): A Tool for Planning and Understanding Tapping and Bimodal AFM Experiments. Beilstein J. Nanotechnol. 2015, 6, 369-379.

(68) Mingeot-Leclercq, M.-P.; Deleu, M.; Brasseur, R.; Dufrêne, Y. F. Atomic Force Microscopy of Supported Lipid Bilayers. Nat. Protoc. 2008, 3, 1654-1659.

(69) Basu, A.; Karmakar, P.; Karmakar, S. Supported Planar Single and Multiple Bilayer Formation by DOPC Vesicle Rupture on Mica Substrate: A Mechanism as Revealed by Atomic Force Microscopy Study. J. Membrane Biol. 2020, 253, 205-219.

(70) Redondo-Morata, L.; Losada-Pérez, P.; Giannotti, M. I. Lipid Bilayers: Phase Behavior and Nanomechanics. Current Topics in Membranes; Elsevier, 2020; Vol. 86, pp 1-55.

(71) Ebeling, D.; Eslami, B.; Solares, S. D. J. Visualizing the Subsurface of Soft Matter: Simultaneous Topographical Imaging, Depth Modulation, and Compositional Mapping with Triple Frequency Atomic Force Microscopy. ACS Nano 2013, 7, 10387-10396.

(72) Rüttiger, C.; Appold, M.; Didzoleit, H.; Eils, A.; Dietz, C.; Stark, R. W.; Stühn, B.; Gallei, M. Structure Formation of Metallopolymer-Grafted Block Copolymers. Macromolecules 2016, $49,3415-3426$.

(73) Dietz, C. Sensing In-Plane Nanomechanical Surface and Sub-Surface Properties of Polymers: Local Shear Stress as Function of the Indentation Depth. Nanoscale 2018, 10, 460468.

(74) Eichhorn, A.L.; Dietz, C. Simultaneous Deconvolution of In-Plane and Out-of-Plane Forces of HOPG at the Atomic Scale under Ambient Conditions by Multifrequency Atomic Force Microscopy. Adv. Mat. Interfaces 2021, 8, 2101288. 
(75) González-Domínguez, I.; Gutiérrez-Granados, S.; Cervera, L.; Gòdia, F.; Domingo, N. Identification of HIV-1-Based Virus-like Particles by Multifrequency Atomic Force Microscopy. Biophysical Journal 2016, 111, 1173-1179.

(76) Garrett, J. L.; Leite, M. S.; Munday, J. N. Multiscale Functional Imaging of Interfaces through Atomic Force Microscopy Using Harmonic Mixing. ACS Appl. Mater. Interfaces 2018, $10,28850-28859$.

(77) Naitoh, Y.; Turanský, R.; Brndiar, J.; Li, Y. J.; Štich, I.; Sugawara, Y. Subatomic-Scale Force Vector Mapping above a $\mathrm{Ge}(001)$ Dimer Using Bimodal Atomic Force Microscopy. Nat. Phys. 2017, 13, 663-667.

(78) Tan, X.; Guo, D.; Luo, J. Dynamic Friction Energy Dissipation and Enhanced Contrast in High Frequency Bimodal Atomic Force Microscopy. Friction 2021.

(79) Garcia, R. \& Perez, R. Dynamic Atomic Force Microscopy Methods. Surf. Sci. Rep. 2002, 47, 197-301.

(80) Lozano, J. R.; Kiracofe, D.; Melcher, J.; Garcia, R.; Raman, A. Calibration of Higher Eigenmode Spring Constants of Atomic Force Microscope Cantilevers. Nanotechnology 2010, 21,465502 .

(81) Labuda, A.; Kocun, M.; Lysy, M.; Walsh, T.; Meinhold, J.; Proksch, T.; Meinhold, W.; Anderson, C.; Proksch, R. Calibration of Higher Eigenmodes of Cantilevers. Rev. Sci. Instrum. 2016, 87, 073705 . 


\section{FIGURES}

$1^{\text {st }}$ Mode

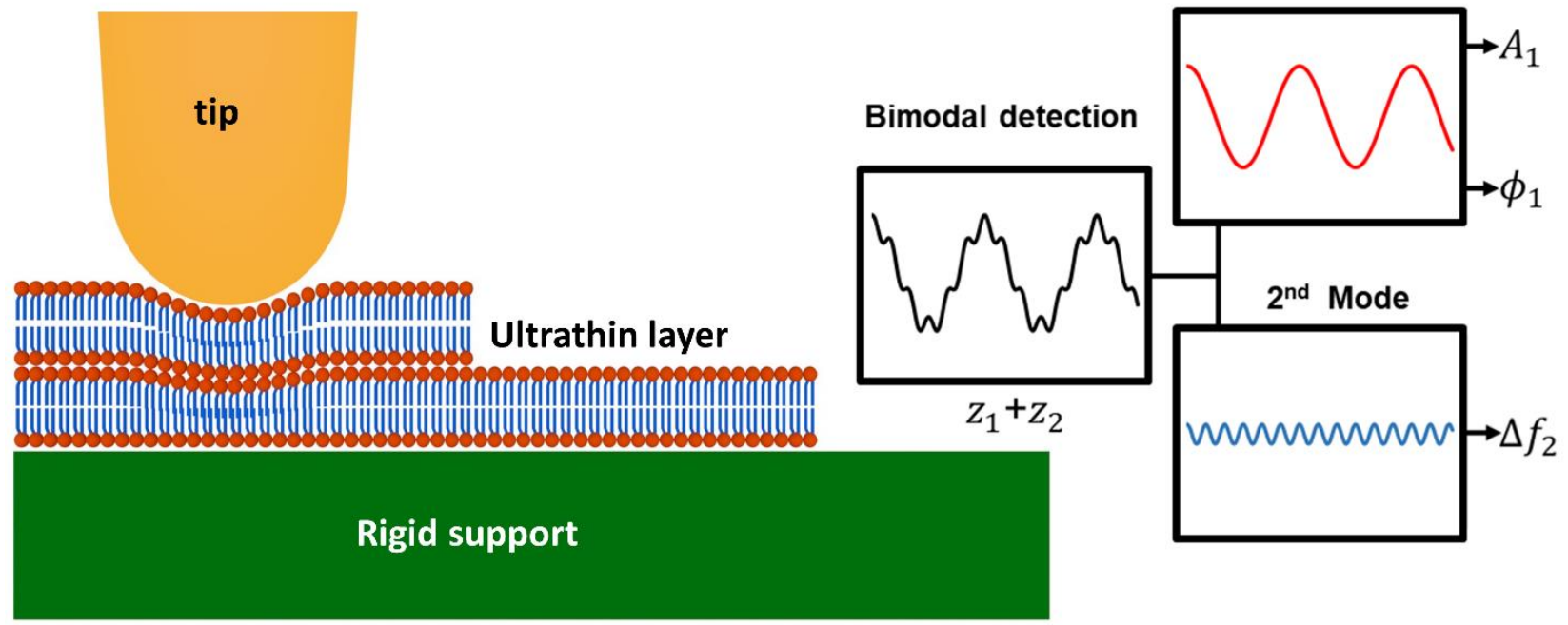

Figure 1. Scheme of a tip-ultrathin layer-solid support interface. The layer depicts a single and two lipid bilayers system. The right panel depicts the detection signals in bimodal AFM (AM-FM configuration). 

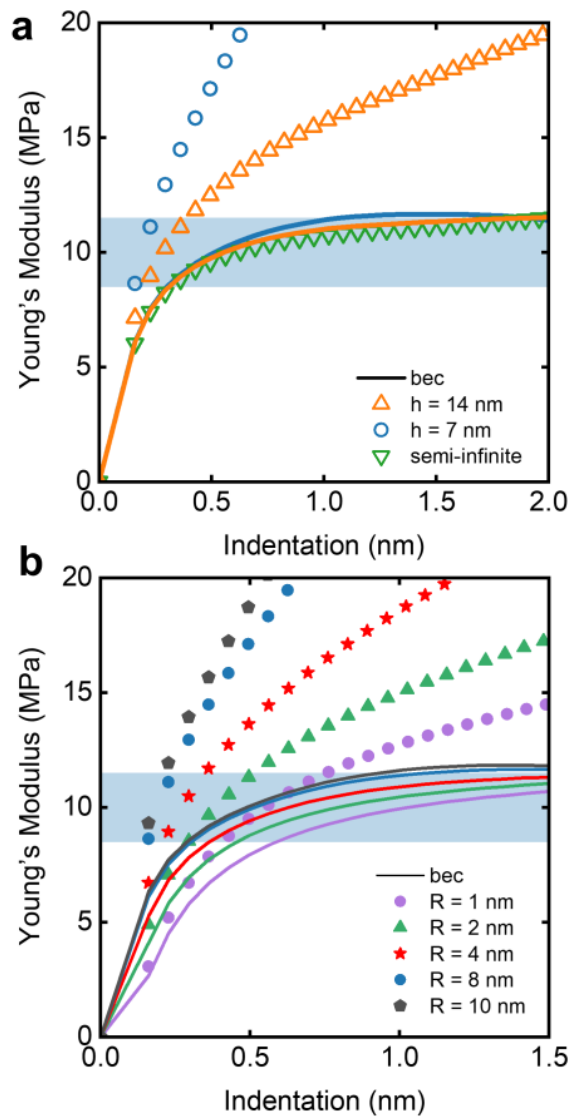

Figure 2. Simulations of bimodal AFM operation for finite thickness and semi-infinite materials.

(a) Young's modulus as a function of the indentation for three different sample thickness $(R=8$ nm). (b) Dependence on the tip's radius for a layer $h=7 \mathrm{~nm}$. The solid lines are the results obtained by using the bottom-effect correction (bec). The symbols correspond to the results given by using the semi-infinite model. The stripe (shaded) shows the values that lie within a $10 \%$ window from the true Young's modulus of the material (10 MPa). The indentation values that produce a stable bimodal AFM operation are marked. 

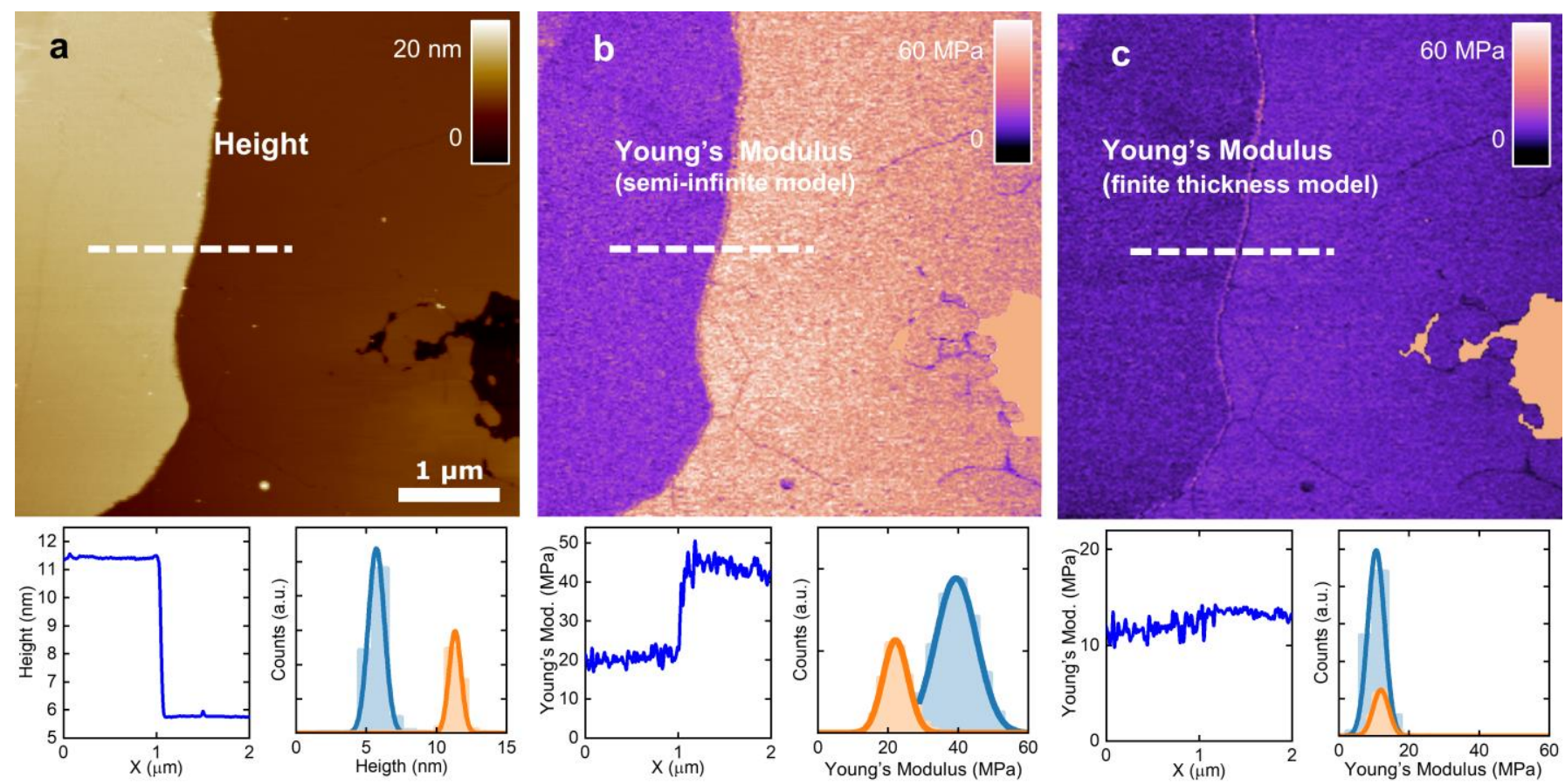

Figure 3. Bimodal AFM topography and elastic modulus maps of a lipid bilayer sample. (a) Topography (top) and height cross-section across the dashed line and height distribution along the map (bottom panels). (b) Young's modulus map obtained by applying a semi-infinite model. (c) Young modulus map obtained by applying a finite thickness model (bottom-effect correction) (top). No differences are observed between the bilayer and the 2-bilayer regions. The bottom panels show the modulus cross-section across the border between a bilayer and a 2-bilayer region and the Young's modulus distribution of the Young's modulus map. The semi-infinite model gives a Young's modulus that depends on the thickness of the number of lipid bilayers. 

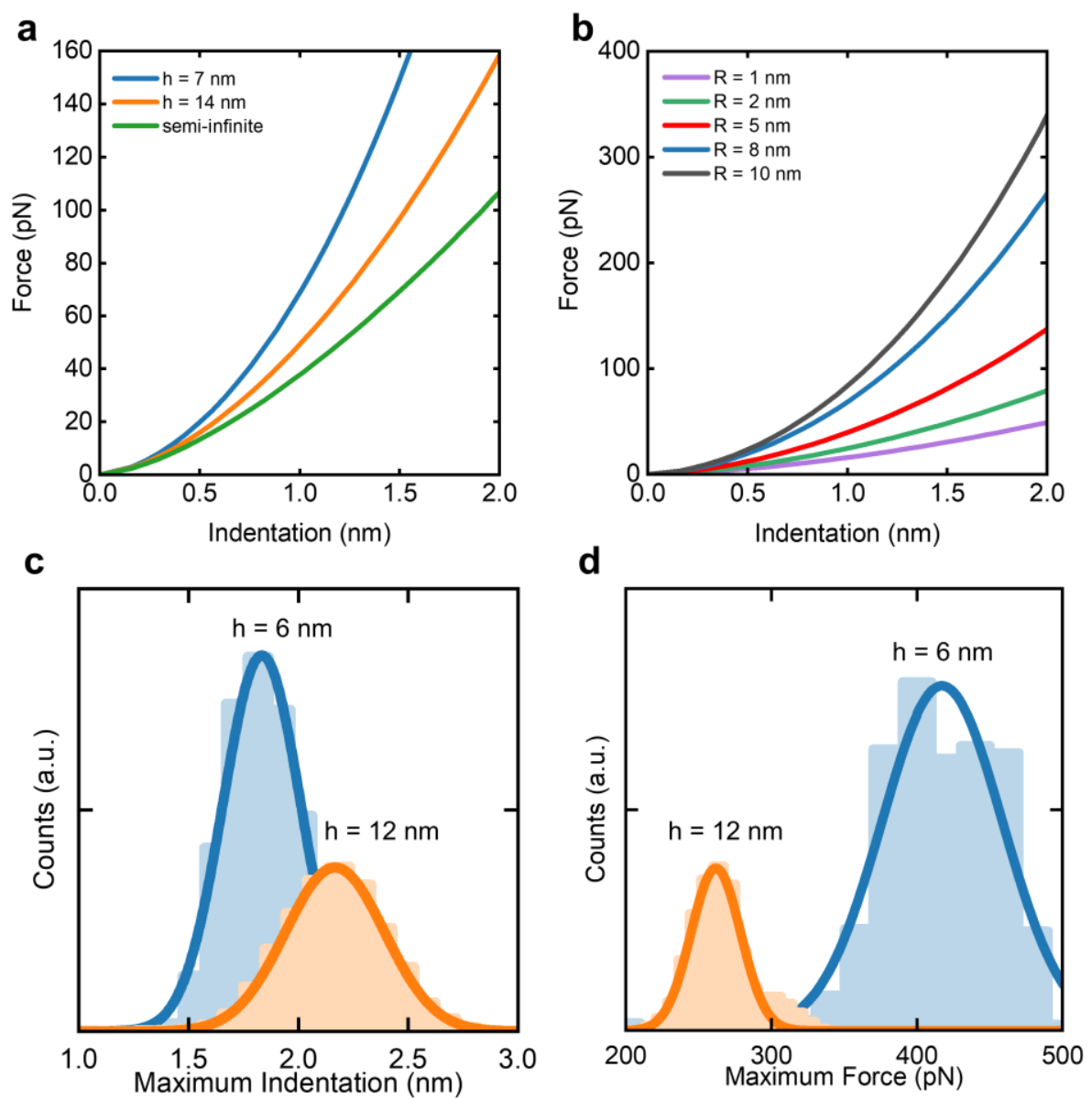

Figure 4. (a) Maximum force applied in bimodal AFM as a function of the indentation for a 10 MPa material (simulation) $R=8 \mathrm{~nm}$. (b) Maximum force applied in bimodal AFM as a function of the indentation for different tips. $E_{s}=10 \mathrm{MPa}$ material; $h=7 \mathrm{~nm}$. (c) Experimental values of the indentation obtained from the different regions of the supported lipid bilayers (from figure 3b). (d) Maximum force exerted on the different regions of supported lipid bilayers (from Fig. $3 b)$. 

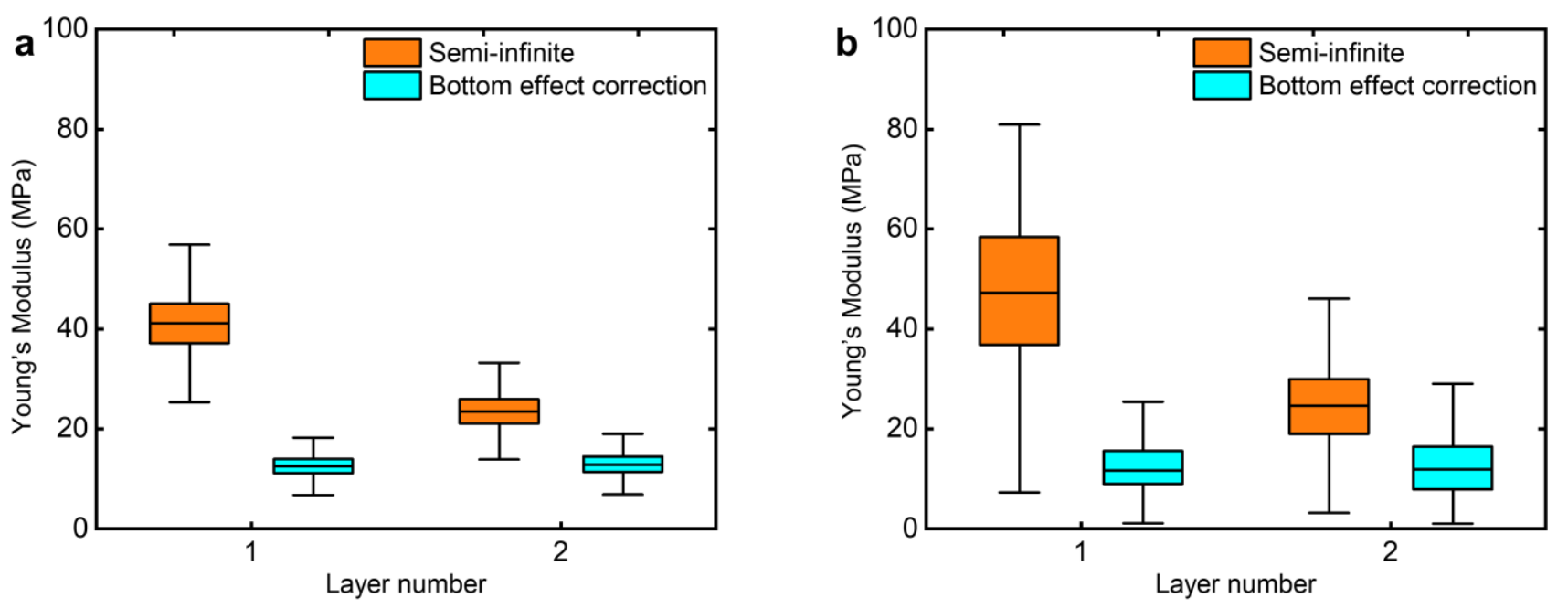

\begin{tabular}{|lll|lll} 
Bimodal & Semi-infinite & Bottom Effect Correction & Force Volume & Semi-infinite & Bottom Effect Correction \\
\hline First Layer & $41.1 \mathrm{MPa}$ & $12.6 \mathrm{MPa}$ & First Layer & $47.2 \mathrm{MPa}$ & $11.6 \mathrm{MPa}$ \\
Second Layer & $23.4 \mathrm{MPa}$ & $12.9 \mathrm{MPa}$ & Second Layer & $24.6 \mathrm{MPa}$ & $11.9 \mathrm{MPa}$
\end{tabular}

Figure 5. Comparison between bimodal and force volume. The measurements were performed on a lipid bilayer system with single and double layer domains. (a) Bimodal AFM. (b) Force volume. 

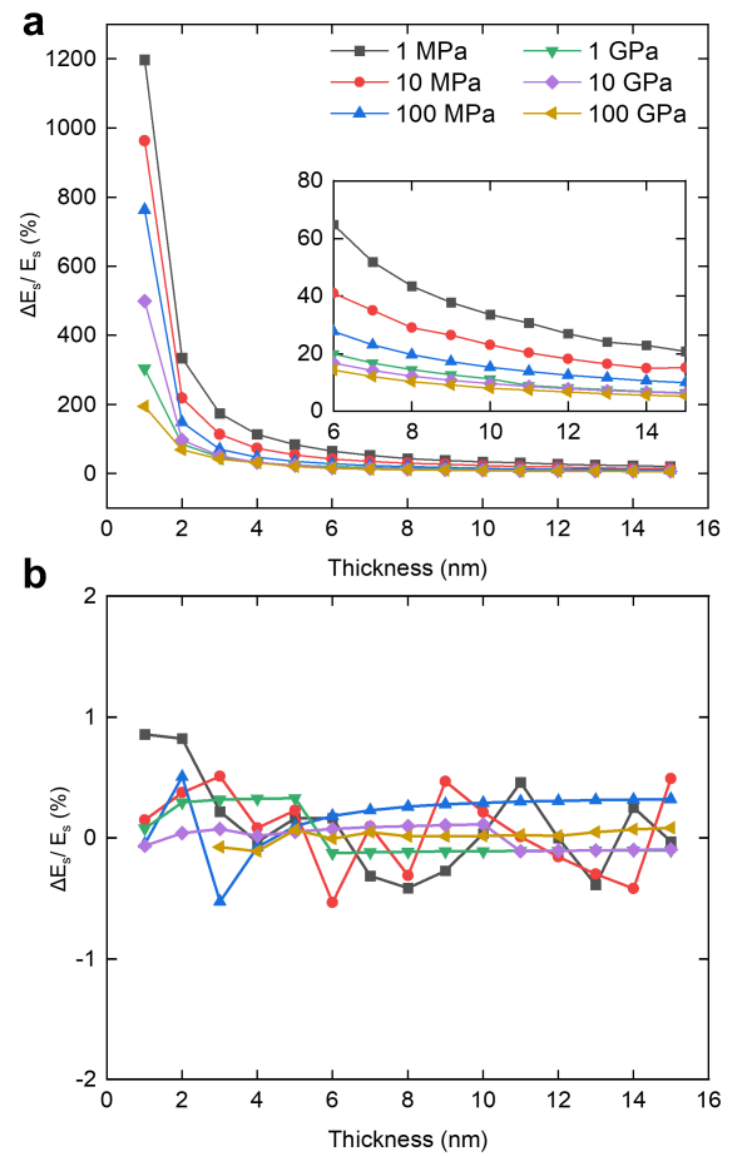

Figure 6. Determination of the Young's modulus of an ultrathin material with bimodal AFM (theory and simulations). The simulations involve layers of different thickness and Young's modulus values. Tip radius $R=2 \mathrm{~nm}$. (a) The use of a semi-infinite contact mechanics model (Hertz, Sneddon) and bimodal theories based on them, leads to significant overestimations of the Young's modulus (relative error $\geq 100-1200 \%$ ). (b) The bottom-effect correction recovers the true Young's modulus of an ultrathin material (1-15 nm) over a 5-orders of magnitude modulus range (1 MPa-100 GPa). The relative error is very small, say below $1 \%$. The fluctuations in the relative error values as a function of the layer thickness $(\leq 0.5 \%)$ are attributed to errors in the numerical integration. The continuous lines are interpolations for visual aid. Details about the simulations are provided in the SI. 